\title{
CHARACTERIZATION OF POLYMER MEMBRANES BY CONTACT ANGLE GONIOMETER
}

\author{
${ }^{1} S z$. Kertész, ${ }^{2}$ T. B. de Freitas, ${ }^{1}$ C. Hodúr \\ ${ }^{1}$ Faculty of Engineering, Univesity of Szeged, Moszkvai krt. 9, 6725, Szeged, Hungary, \\ e-mail: kertesz@mk.u-szeged.hu \\ ${ }^{2}$ Federal Technological University of Paraná Campus Campo Mourão, \\ Via Rosalina M. dos Santos 1233, Campo Mourão, Paraná, Brazil
}

\begin{abstract}
The wider applications of all membrane separation processes have a main obstacle, namely the fouling phenomena, which have to be understood in more details. Surface properties, hydrophilic and hydrophobic characteristics of a polymer membrane can be determined by measuring the contact angle. The hydrophilicity of a membrane has an important influence on its performances, like permeate flux, membrane rejection or membrane fouling characteristics. In our work the contact angles of three kinds of typically commercial ultrafiltration (UF-PES-4), nanofiltration (NE-90) and reverse osmosis (LFC-30) membranes were firstly investigated and compared by contact angle goniometer measurements. The relationships between the contact angles were researched by well considering the effects of membrane sample pretreatments by distilled water prewetting and water droplet volume. Furthermore, the effects of prewetting, water droplet contact time on different molecular weight cut-off ultrafiltration membranes' surface and droplet $\mathrm{pH}$ on the contact angle values were also investigated. Moreover, fresh, clean and dry, as well as pretreated, and fouled UF membranes were also measured and compared.
\end{abstract}

Keywords: contact angle, goniometer, hydrophilicity, polymer membrane

\section{INTRODUCTION}

Membrane separations, microfiltration (MF), ultrafiltration (UF), nanofiltration (NF) and reverse osmosis (RO) have been extensively used in food industry for selective separation of different molecules and compounds. In these membrane processes the pressure difference is the driving force between the two sides of the membrane, therefore they are called pressure-driven membrane processes or membrane separation technologies. For example all of these mentioned processes have been in use in the dairy industry for about 4-5 decades [1]. However, they more widely industrial applications have a main obstacle, namely the membrane fouling or clogging. Fouling phenomena are necessary to understand it in more details. The surface properties of membranes can be characterized by measuring the contact angle. Membrane hydrophilicity or hydrophobicity characteristics can be determined by measuring the contact angles. Low contact angle indicates high water affinity and also, any change in the contact angle can indicate more fouling formation because of differences in surface properties of the deposit and the clean membrane. Those values are related to the surface energy parameters through the Young-Laplace equation by the sessile drop method measurement, which measures the contact angle of the water droplet on the membrane surface in the ambient air [2;3]. Some important parameters can affect the contact angle values of the membrane, like $\mathrm{pH}$, which affects the charge of both the membrane surface and the liquid macromolecules. These charge effects and adsorption propensity, which depends on membrane properties charge, hydrophobicity and porous structure, can cause different membrane materials to display different fouling propensities $[4 ; 5]$. The main objective of our study was to characterize different types of membranes for contact angle measurements, and to understand the effects of membrane materials, molecular weight cutoff and pretreatments techniques on the contact angle values into the details.

\section{MATERIALS AND METHODS}

\subsection{Goniometer}

The contact angle values were measured by an OCA 15 Pro goniometer (DataPhysics, Germany) with DataPhysics SCA software controlled system designed for Microsoft Windows. The contact angles of the membranes were measured in sessile drop method. Before the measurement a Hamilton $500 \mu \mathrm{L}$ syringe had to be filled by the water or solution and fixed a piece of the examined membrane support of 10x40mm dimensions. Then the intensity of the backlight, focus and position of the membrane was arranged. The configurations in the software have to be adjusted, like type of syringe, volume of the droplets, and velocity of dispense. 


\subsection{Membranes and pretreatments}

The new, dry characterized membranes were measured by the above contact angle sessile drop method and then membranes were pretreated by prewetting into distilled water for $24 \mathrm{~h}$ at room temperature. Then it was dried with pressurized air and their contact angles were determined again for investigating the influence of prewetting on the hydrophilicity of the membranes.

For characterized the ultrafiltration membranes 4 and $10 \mathrm{kDa}$ molecular weight cut-off (MWCO) polyethersulfone (PES) membranes and a $30 \mathrm{kDa}$ regenerated cellulose membrane; for nanofiltration a thin-film composite (TFC) NE90 (90 Da) membrane and for reverse osmosis a $30 \mathrm{Da}$ LFC thin-film composite membrane were used. The volume of solution or water droplet for the contact angle measurement was increased from $1 \mu \mathrm{L}$ to $20 \mu \mathrm{L}$ for time dependence experiments. All of the measurements were repeated 5 times with each membrane, to calculate an accurate average.

\subsection{Contact angle measurements}

To measure the time dependence of the contact angle values $10 \mu \mathrm{L}$ of droplet volume was used. The contact angle was measured immediately after the dropping and at intervals of 10 seconds. To measure the effect of fouling on membrane $10 \mu \mathrm{L}$ of droplet volume was also used. A clean and two differently fouled $30 \mathrm{kDa}$ regenerated cellulose membranes were tested and compared. The fouled membranes were produced by the ultrafiltration concentration experiments of model dairy wastewater $\left(\mathrm{c}_{\text {milk powder }}=5 \mathrm{~g} / \mathrm{L} ; \mathrm{c}_{\text {detergent }}=0.5 \mathrm{~g} / \mathrm{L}\right)$ till volume reduction ratio (VRR) of 5 after ultrasonic pretreatment (at $30 \mathrm{~min}$ of retention time a $200 \mathrm{~W}$ of ultrasound energy power for treating half liter wastewater was used) and without ultrasonic pretreatment. To analyze the $\mathrm{pH}$ effect on contact angle, CL- 80 anionic detergent was used to prepare standard distilled water $\mathrm{pH}$ solutions from $\mathrm{pH} 7$ to 12 .

\section{RESULTS AND DISCUSSIONS}

In Fig. 1 the contact angles of three kinds of typically fresh, new and dry, commercial polymer membranes (UF: 4 kDa PES; NF: 90 Da TFC; RO: 30 Da TFC), were firstly investigated and compared by contact angle goniometer measurements. The results showed that the increase of droplet volumes from 1 to $20 \mu \mathrm{L}$ had no significant impact on the results. For a comparison, the values of average contact angle were equal to $64^{\circ}, 10^{\circ}, 49^{\circ}$ for UF, NF and RO membranes, respectively. The three different types of membrane presented hydrophilic characteristics because the measured values of the contact angles were always below $90^{\circ}$. However the differences between the membrane types were considerable and NF membrane was observed as the most hydrophilic membrane with the lowest contact angle results.

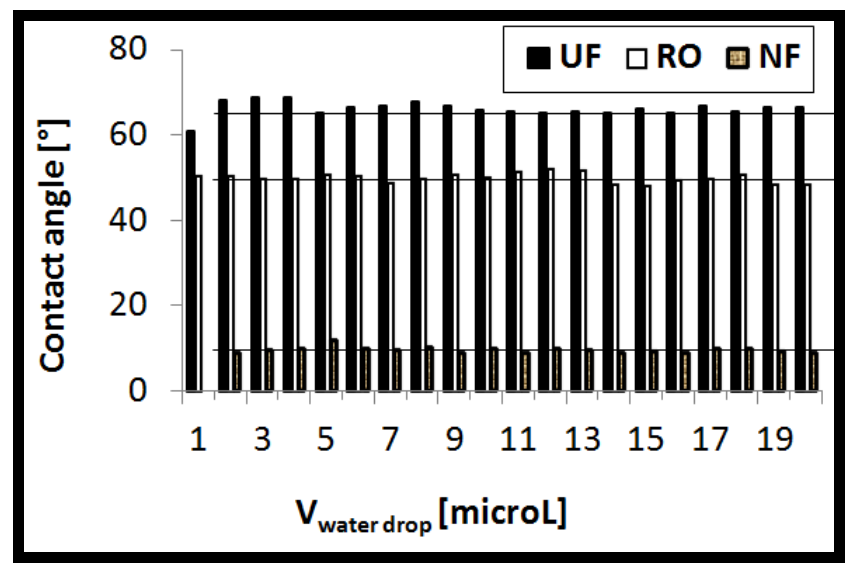

Figure 1. Influence of water droplet volume on contact angles

Fig. 2 presented the effects of pretreatment with water prewetting on the hydrophilicity of the polymer membranes. For a comparison, the prewetting of the membranes increased the hydrophilicity with decreasing of the contact angles by $0.4 \% 34.4 \%, 12 \%$, for UF, NF and RO membranes, respectively. It suggests that the prewetting resulted more hydrophilic membrane surface and lower fouling tendency in 
each case. The lowest difference was observed using UF membranes, which was not significant, and the highest difference had the NF membrane.

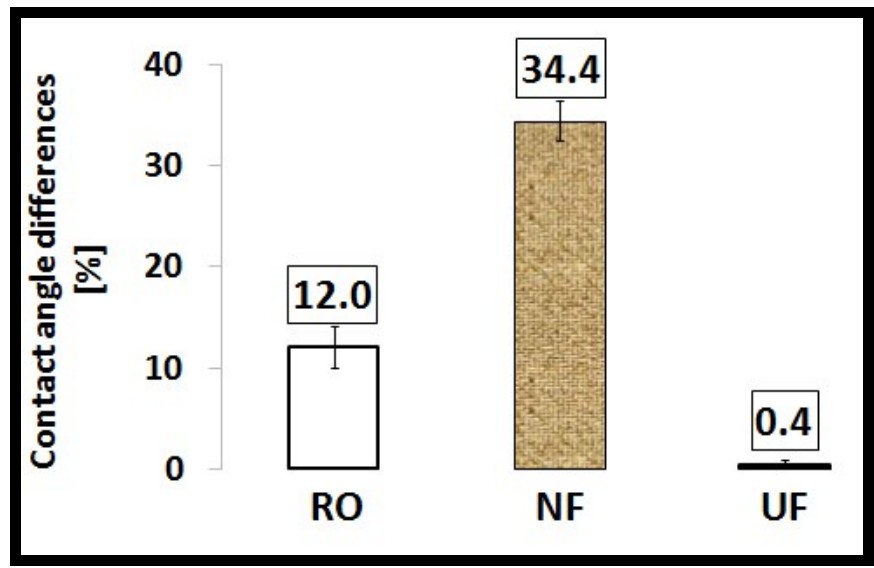

Figure 2. Percentage differences in contact angles between dry and pretreated membranes

The time dependence of contact angles on the surface of higher MWCO membranes can be quite important, since the values could change as a function of wettability. To measure this phenomenon prewetted 4 and 10 $\mathrm{kDa}$ PES membranes were analyzed and shown in Fig. 3. The contact angle decreased from $49.2^{\circ}$ to $41.2^{\circ}$ (which means $16.3 \%$ decreasing rate) and from $43^{\circ}$ to $37.7^{\circ}(12.3 \%$ decreasing rate) testing $4 \mathrm{kDa}$ and 10 kDa UF PES membranes, respectively. In the case of NF and RO membranes the contact angle changing as a function of time was not significant due to the smaller MWCO. However, the UF MWCO is important to change the time dependence of the contact angles, the polymer type of the membrane is also important parameter. From our experiments it was obvious that regenerated cellulose UF membrane did not have effect on the time dependence of the contact angles even if it had higher $30 \mathrm{kDa}$ MWCO compare to the (4 and $10 \mathrm{kDa}$ ) PES membranes.

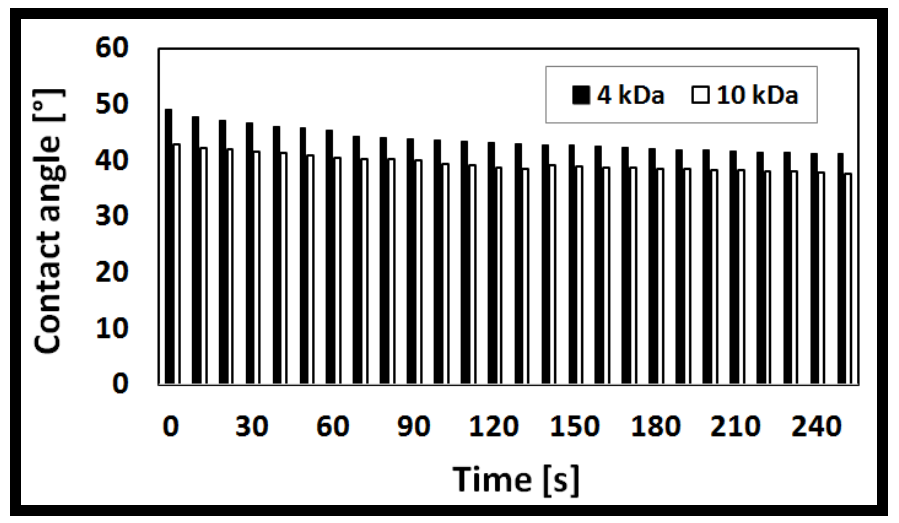

Figure 3. Time dependence of contact angle values on pretreated 4 and $10 \mathrm{kDa}$ polyethersulfone membranes

In Fig. 4 the contact angles of a fresh, clean and dry membrane and two differently fouled $30 \mathrm{kDa}$ MWCO regenerated cellulose membranes are shown. The fouled membranes were analyzed after ultrafiltration concentration experiments of model dairy wastewater with and without ultrasonic pretreatment, with the same parameters as we discussed in Materials and methods. Based on the results it could be concluded that the fresh membrane had the most hydrophilic surface and the fouled membranes had higher contact angles. Moreover, the ultrasonically pretreated wastewater ultrafiltration resulted the highest contact angle $\left(75.9^{\circ}\right)$, which indicates that the ultrasound pretreatment resulted higher membrane fouling, due to the ultrasound molecular degradation effect. 


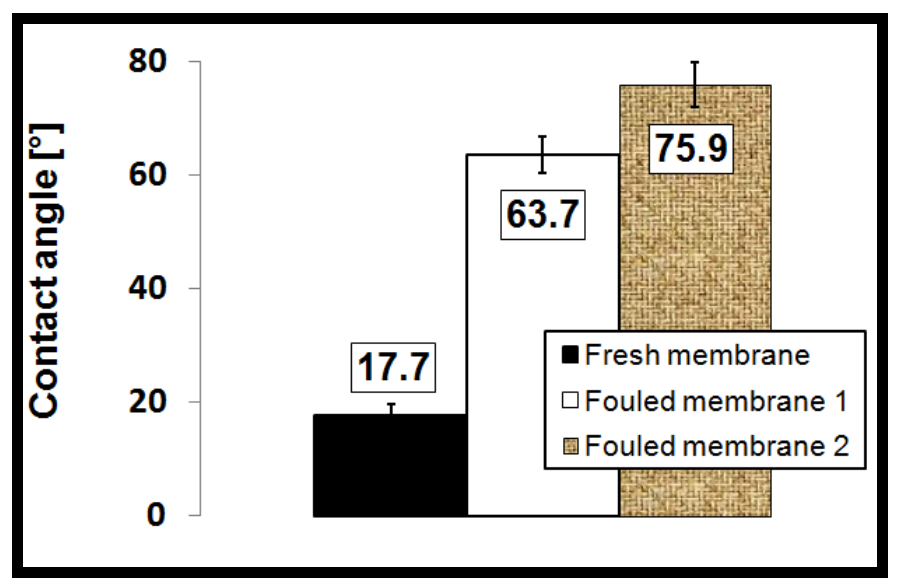

Figure 4. The contact angles of a clean (Fresh membrane) and two differently fouled $30 \mathrm{kDa}$ regenerated cellulose membranes (Fouled membranes were analyzed after ultrafiltration concentration experiments of model dairy wastewater with (2) and without (1) ultrasonic pretreatment)

In Fig. 5 the contact angles as a function of $\mathrm{pH}$ are shown. The dry $30 \mathrm{kDa}$ MWCO regenerated cellulose UF membrane in contact with the different detergent $\mathrm{pH}$ solutions changed the wettability of the membrane surface. The contact angles decreased from $63.3^{\circ}$ to $22.1^{\circ}$ as the $\mathrm{pH}$ value increased from 7 to 12 . From our results we can conclude that the alkaline $\mathrm{pH}$ resulted higher membrane hydrophilicity, since the surface features were changed significantly.

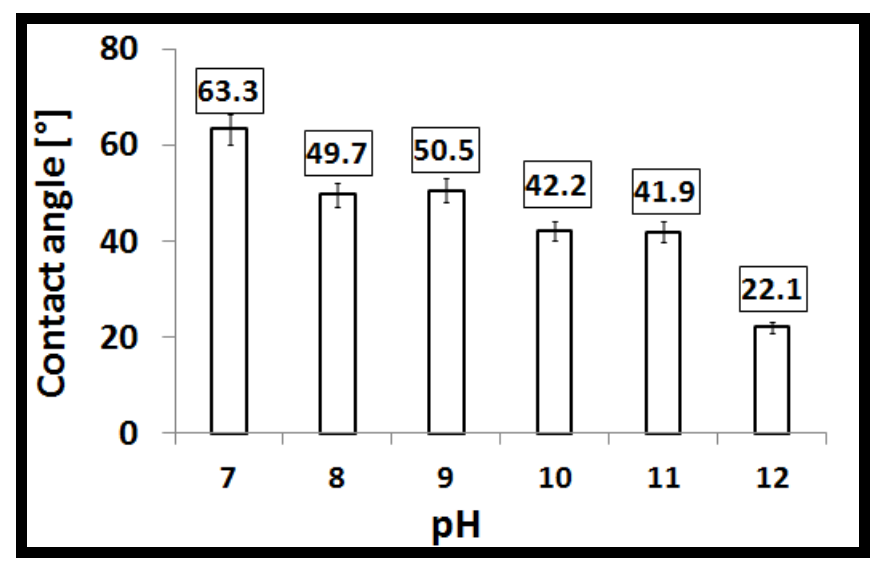

Figure 5. Contact angle values versus pH increasing

\section{CONCLUSION}

From the water droplet volume changing experiments we can conclude that the increase of droplet volumes had no significant impact on the contact angle values. Compare to the results of UF, NF and RO membranes the differences between them were considerable and NF membrane was observed as the most hydrophilic membrane. The membrane pretreatment experiments with water prewetting showed us that the pretreatment resulted more hydrophilic membrane surface and lower fouling tendency in each case. The lowest contact angle difference was observed with UF membranes, as well as NF membranes showed the highest difference. Our time dependence of contact angle experiments suggested that the UF MWCO was important to change the time dependence of the measuring, but the polymer type of the membrane was also an important parameter. It was obvious that the regenerated cellulose UF membrane did not have effect on the time dependence of the contact angles even if it had higher $30 \mathrm{kDa}$ MWCO compare to the 4 and 10 $\mathrm{kDa}$ PES membranes. Based on the results of the fouled membranes experiments it can be concluded that the fresh membrane had the most hydrophilic surface and the fouled membranes had higher contact angles. Moreover, the ultrasonically pretreated wastewater ultrafiltration resulted the highest contact angle, which indicated that the ultrasound pretreatment resulted higher membrane fouling, due to the ultrasound 
molecular degradation effect. Finally, the $\mathrm{pH}$ experiments proved that the alkaline $\mathrm{pH}$ resulted higher membrane hydrophilicity, due to the significantly changed membrane surface features.

\section{REFERENCES}

[1] Marella, C., Muthukumarappan, K., Metzger, L.E. (2013), Application of Membrane Separation Technology for Developing Novel Dairy Food Ingredients, Journal of Food Processing \& Technology, 4 (9), 269-274

[2] Gourley, L., Britten, M., Gauthier, S.F., Pouliot, Y. (1994), Characterization of adsorptive fouling on ultrafiltration membranes by peptides mixtures using contact angle measurements, Journal of Membrane Science, 97, 283-289

[3] Balcioglu, G., Gönder, Z.B. (2014), Recovery of baker's yeast wastewater with membrane processes for agricultural irrigation purpose: Fouling characterization, Journal of Membrane Science, 255, 630-640

[4] Sutzkover-Gutman, I., Hasson, D., Semiat, R, (2010), Humic substances fouling in ultrafiltration processes, Journal of Membrane Science, 261, 218-231

[5] Li, Q., Pan, X., Qu, Z., Zhao, X., Jin, Y., Dai, H., Yang, B., Wang, X. (2013), Understanding the dependence of contact angles of commercially RO membranes on external conditions and surface features, Desalination, 309, 38-45 